\title{
A Study on Characteristics of Flow Field of Air Gap in Maglev Spherical Active Joints for Multi-DOF Spindle System
}

\author{
Fan ZHANG*, Li ZENG**, Yan LI***, Zi ZENG****, Zhida ZHU*****, Jin SUN******, \\ Min DAI******** \\ *School of Mechanical Engineering, Yangzhou University, Yangzhou225127, China, E-mail: fzhang@yzu.edu.cn \\ **School of Mechanical Engineering, Yangzhou University, Yangzhou225127, China, E-mail: lizengcf@163.com \\ ***School of Mechanical Engineering, Yangzhou University, Yangzhou225127, China, E-mail: yli@yzu.edu.cn \\ ****School of Mechanical Engineering, Yangzhou University, Yangzhou225127, China, E-mail: zzeng@yzu.edu.cn \\ *****School of Mechanical Engineering, Yangzhou University, Yangzhou225127, China, E-mail: zdzhu@yzu.edu.cn \\ ******School of Mechanical Engineering, Yangzhou University, Yangzhou225127, China, E-mail: jinsun@yzu.edu.cn \\ *******School of Mechanical Engineering, Yangzhou University, Yangzhou225127, China, E-mail: mdai@yzu.edu.cn
}

cross $^{\text {ref }}$ http://dx.doi.org/10.5755/j01.mech.24.5.19492

\section{Introduction}

Spindle tools capable of six-degree-of-freedom spatial movement in any posture is one of the key features of existing advanced CNC machine tools. The multi-degreeof-freedom spindle systems are generally classified into two types: new spindle with parallel structure and traditional spindle with cascaded structure [1]. The former changes its position and posture by employing several servo-motor or hydraulically driven links with controllable lengths, whereas the latter utilizes multiple mechanical rotating structures, each of which controls the rotation for only one degree-of-freedom, thus controlling the direction of spindle tool (Fig. 1).Both types of spindles are faced with the following defects: 1 . numerous joints complicates the mechanical structure and worsens the driving efficiency and reliability; 2 . serious abrasion caused on the friction surfaces of joints response speed and dynamic characteristics; and 3. accumulation of mechanical errors resulting in lowering the accuracy of control system.

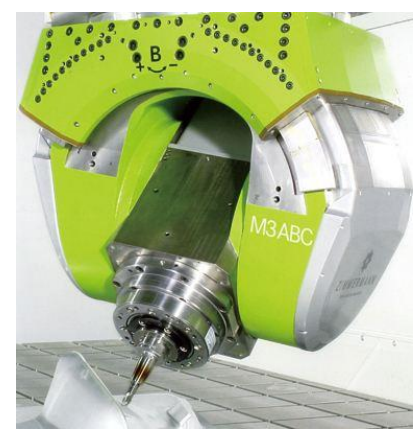

a

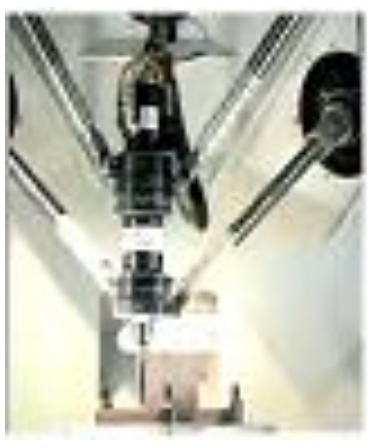

b
Fig. 1 Multi-DOF CNC machines: a - spindle with cascaded structure, $\mathrm{b}$ - spindle with parallel structure

To address these defects, quasi-human joints with better efficiency could be constructed based on simplified spherical joints, that is to say to utilize maglev spherical active joints supported and driven by motors with spherical surface in contact [2-7]. Using such kind of active joints in a CNC machine will reduce the complexity of multi-DOF spindles and improve their position and posture accuracies and response speed. Under high-speed operation of spindles, rotor surface drives the air in gap to rotate along with the rotor and form a circumferential air circulation in gap, thereby engendering an air film in the flow field of air gap between the stator and the rotor to generate a levitating force or resistance [8]. For the film, how its flow field pressure and dynamic characteristics are distributed and changed exerts direct influence upon the dynamic and static performance of joint. However, present studies normally ignore the effects of the levitating force or resistance generated by the air film upon rotor's movement and dynamic performance, which explains the deviation between theoretical analysis and reality in terms of their motions. A lot of scholars [9-16] have achieved some valuable results in both terms of theoretical modeling and experimental verification, which have been practiced in engineering. However, studies on the characteristics of flow field of air gap in maglev spherical active joint have been little reported in both China and other countries. This paper explored the flow field of air gap in maglev spherical active joint and its dynamic \& static characteristics based on aerodynamics, established mathematical models for dynamic \& static pressures and kinetic parameters of flow field of air gap, and, with simulation and experiment, investigated how the flow field of air gap impacts its static load-carrying capacity and dynamic characteristics.

\section{Principles of maglev spherical active joints and variation pattern of air gap}

\subsection{Principles of joint generating electromagnetic torque and levitating force}

However, the difference from conventional motors is that, the joints should extend its rotor from uni-dimensional rotation around a fixed axis to spatial rotation around a fixed point. In this case, rotor's angular velocity vector $\omega$ has to be changed from a directed scalar to a spatial vector in an arbitrary direction. $\boldsymbol{\omega}=\omega_{x} \boldsymbol{i}+\omega_{y} \boldsymbol{j}+\omega_{z} \boldsymbol{k}$, where $\boldsymbol{i}, \boldsymbol{j}$ and $\boldsymbol{k}$ represent unit vectors in three arbitrary axes, respectively. However, nonlinear coupling relations are shown among mechanical equilibrium equations of three orthogonal axes. Thus, to effectively and separately manipulate the three vector components of angular velocity for the rotor of joint, three axial electromagnetic torques $M_{x}, M_{y}$ and $M_{z}$, which 
are independently controllable. In an orthogonal coordinate system, the three torque vector components are combined as $\boldsymbol{M}=M_{x} \boldsymbol{i}+M_{y} \boldsymbol{j}+M_{z} \boldsymbol{k}$, and the resultant torque is a spatial vector in an arbitrary direction.

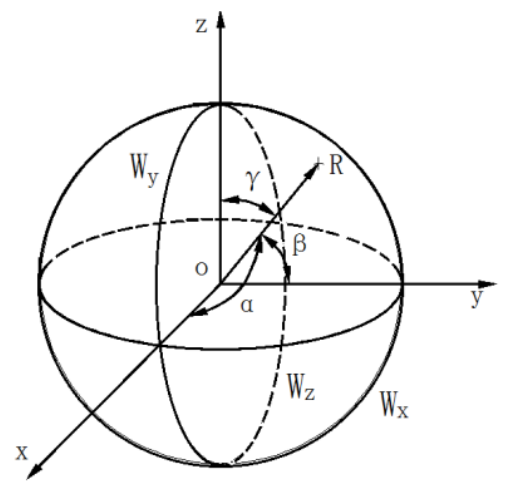

Fig. 2 Coordinate system of winding

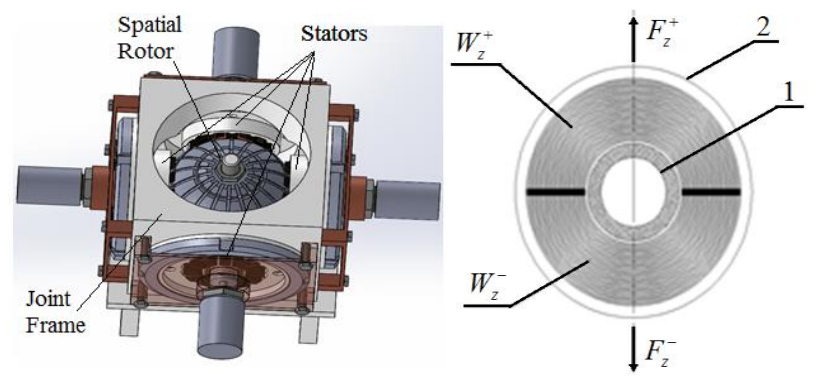

Fig. 3 3D model of joint Fig. 4 Generation of levitastructure tion in $\mathrm{z}$-axis

To yield electromagnetic torques in arbitrary controllable spatial directions, the maglev spherical active joint should produce a resultant magnetic field, whose amplitude and axis direction can totally be manipulated. Based on a Cartesian coordinate system, this paper prepared threephase AC windings around each coordinate axis. A two-dimensional magnetic field vector was formed by electrifying symmetrical three-phase windings with symmetrical threephase alternating currents, and the magnetic field vector rotated round its axis. These magnetic field vector could also be formed by two phase AC windings with two quadrature alternating currents. Then combining 2D magnetic field vectors of all 3 coordinate axes with each other formed a 3D magnetic field, which rotated around an arbitrary axis passing through a fixed spatial point. As shown in Fig. 2, three windings that generated 2D magnetic fields are represented by $W_{x}, W_{y}$ and $W_{z}$, respectively. Therefore, the structure of a maglev spherical active joint can be designed according to the formation mechanisms of a spatial rotating magnetic field, as shown in Fig. 3. The core of stator also provided a spherical surface internally to accommodate to the spherical rotor and engender a uniform air gap between them.

Three windings (corresponding to three coordinate axes) with axes mutually orthogonal were also embedded into the slotted core of stator. Each winding was divided into two sections, which were symmetrically wrapped around the core of stator in two hemispherical slots. Fig. 4 shows the electromagnetic winding in $\mathrm{z}$ direction of the rotor of maglev spherical active joint, where $W_{z}$ is divided into two symmetrical windings $W_{z}^{+}$and $W_{z}^{-}$, representing the posi- tive and negative hemispherical windings of stator, respectively, with 1 and 2 referring to the spherical rotor and stator of joint, respectively. Windings wrapped around 3 axes were energized by adjustable 3 -phase alternating currents $i_{A}$, $i_{B}$ and $i_{C}$ and a controller was employed to control torques and speeds (or angular displacements) for both winding sections on each axis, thereby generating parallel electromagnetic torques with same direction to drive the rotor to rotate around this axis. For example, providing the generated resultant electromagnetic torque in $\mathrm{z}$-axis was $M_{z}=M_{z}^{+}+M_{z}^{-}$. The levitated rotor shares the same working principles with those traditional electromagnet bearings. As illustrated in Fig. 4, when the rotor in $z$ direction deviated from its balanced position under interference, its gap in positive $\mathrm{z}$-axis shrank. For such conditions, to return the rotor back to stator's centre (that's the balanced position) under closed-loop control, the currents applied onto the hemispherical windings of joint's stator below the rotor were increased by $\Delta i_{z}$, whereas the currents applied onto other side were reduced by $\Delta i_{z}$. The difference between electromagnet pulls generated by upper and lower hemispherical windings of stator enabled the rotor to return to the balanced position at the centre of stator and the resultant levitating force was $F_{z}=F_{z}^{+}-F_{z}^{-}$, as shown in Fig. 4 . The rotation and levitation mechanisms in $\mathrm{x}$-axis and $\mathrm{y}$-axis can be analysed in the same way.

\subsection{Variation pattern of air gap in maglev joint}

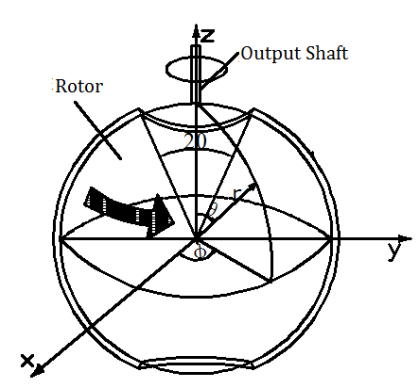

a

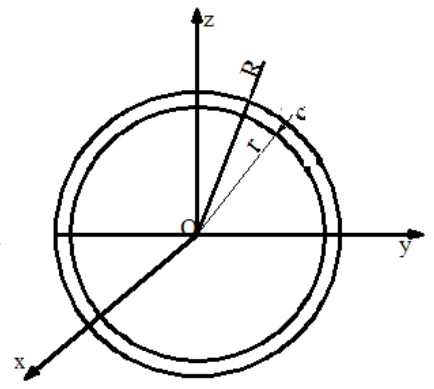

b

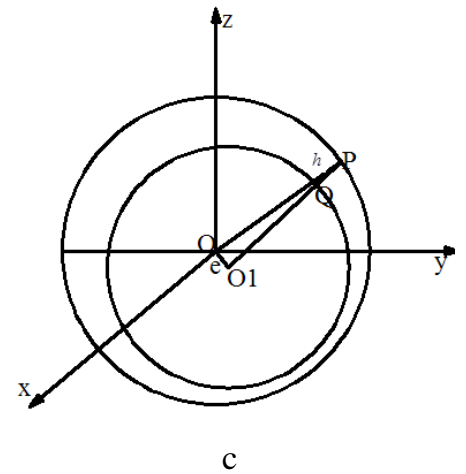

Fig. 5 A 3D spherical coordinate system for flow field of air gap in a maglev spherical active joint: a - 3D coordinate system of active joint, $b$ - the air gap without deviation of the ball center, $\mathrm{c}$ - the air gap with deviation of the ball center

When the rotor of maglev spherical active joint rotates at a high speed around the spindle, the air circulation in the flow field of air gap can directly affect the dynamic \& 
static performances of joint. The characteristics of air circulation are in close relation with the variation pattern of air gap length. As shown in Fig. 5, a, a 3D coordinate system was established for the maglev spherical active joint.

The air gap is illustrated in Fig. 5, b when there is no deviation for the centre of sphere of rotor, where $r$ is the outer radius of rotor; $R$ is the inner radius of stator; and $c$ is the average length of air gap when the centre of sphere of stator coincides with the centre of sphere of rotor. Fig. 5, c shows the case where the centre of sphere of rotor deviates from the balanced position, where $h$ is the air gap length between stator and rotor at arbitrary spatial positions.

$$
h_{11}(t)=x(t)-m_{11}(t)
$$

Radius vector $\boldsymbol{R}$ in the coordinate system $O x y z$ is represented as [17]17:

$$
R=i \sin \theta \cos \varphi+j \sin \theta \sin \varphi+k \cos \theta
$$

The eccentricity from the geometrical centre of rotor $O_{1}$ to the geometrical centre of stator $O$ is represented as:

$$
\overrightarrow{\boldsymbol{e}}=\boldsymbol{i} \cdot x+\boldsymbol{j} \cdot y+\boldsymbol{k} \cdot z,
$$

Take the geometrical centre of rotor $O_{1}$ as the origin of a radius vector, which intersects with the internal surface of stator at point P. As the eccentricity $e$ is far less than $r$ and $R, \angle O_{1} P O$ is little and it can be obtained that $\angle O_{1} P O \approx 1$, so in the triangle as shown in the figure, the relation is as follows :

$$
h+r=R-\vec{e} \cdot \boldsymbol{R}
$$

or

$$
h=c-x \sin \theta \cos \varphi-y \sin \theta \sin \varphi-z \cos \theta
$$

\section{Static load-carrying capacity model for flow field of air gap in maglev joint}

3.1. Static pressure distribution model for air film in air gap of maglev joint.

When the rotor rotates at a high speed, the air in gap will form a layer of air film against the stator. The static pressure distribution on the air film can be derived from continuity equation of fluid, equation of motion, state equation of fluid and energy equation. A pressure distribution equation can also be derived for the compressive flow field in a spherical coordinate system and its dimensionless form is expressed as follows:

$$
\frac{\partial}{\partial \varphi}\left(H^{3} \cdot \frac{\partial P^{2}}{\partial \varphi}\right)+\sin \theta \frac{\partial}{\partial \theta}\left(H^{3} \cdot \sin \theta \cdot \frac{\partial P^{2}}{\partial \theta}\right)=\sin ^{2} \theta \cdot \Lambda \cdot \frac{\partial(P H)}{\partial \varphi}+2 \sin ^{2} \theta \cdot \Lambda \cdot \frac{\partial(P H)}{\partial \tau}
$$

where: $\Lambda$ is the load-carrying coefficient, $\Lambda=\frac{12 \mu \omega}{p_{a}}\left(\frac{r}{c}\right)^{2}$; $\mu$ is the dynamic viscosity of fluid; $r$ is the radius of joint's rotor; $P$ is the dimensionless pressure of flow field and $P=p / p_{a} ; p_{a}$ is the ambient atmospheric pressure $(P a) ; H$ is the dimensionless thickness of air film, $H=\frac{h}{c}=1-\varepsilon_{x} \sin \theta \cos \varphi-\varepsilon_{y} \sin \theta \sin \varphi-\varepsilon_{z} \cos \theta, \varepsilon_{x}=\frac{x}{c}$, $\varepsilon_{y}=\frac{y}{c}, \varepsilon_{z}=\frac{z}{c}, \tau=\omega t, t$ is the time and $\omega$ is the circular frequency for rotor working.

\subsection{Static load-carrying capacity of air film in air gap of maglev joint}

As the static pressure distribution equation of air film has no relation with the time, each of following static variables is represented with a subscript 0 for the purpose of being distinguished. So according to Eq. (6), the static pressure distribution equation we can obtain is as follows:

$$
\begin{gathered}
\frac{\partial}{\partial \varphi}\left(H_{0}{ }^{3} \frac{\partial P_{0}^{2}}{\partial \varphi}\right)+\sin \theta \cos \theta\left(H_{0}{ }^{3} \frac{\partial P_{0}^{2}}{\partial \theta}\right)+\sin ^{2} \frac{\partial}{\partial \theta}\left(H_{0}{ }^{3} \frac{\partial P_{0}^{2}}{\partial \theta}\right)=\sin ^{2} \theta \cdot \Lambda \cdot \frac{\partial}{\partial \varphi}\left(P_{0} H_{0}\right), \\
H_{0}=\frac{h_{0}}{c}=1-\varepsilon_{x 0} \sin \theta \cos \varphi-\varepsilon_{y 0} \sin \theta \sin \varphi-\varepsilon_{z 0} \cos \theta
\end{gathered}
$$

Then solve for the boundary conditions: 1) At the place where the air gap contacts with the atmosphere, $P_{0}=P_{a}$; 2) The rotor has an eccentric angle $\varphi_{0}=\arctan \left(\varepsilon_{y} / \varepsilon_{x}\right)$, and let $P_{0}=P_{a}$ at the place where $\varphi=\arctan \left(\varepsilon_{y} / \varepsilon_{x}\right)+\pi$.

We can combine Eq. (7) and Eq. (8) to get the thickness $H_{0}$ and the pressure $P_{0}$ of air film. Further, after we obtain the steady state, the load on the static air film and the damping moment of air film with the direction along the axis of rotation can be expressed as:

$$
\left\{\begin{array}{l}
F_{x 0}=-\iint P_{0} \sin \varphi \cos \theta d \Omega \\
F_{y 0}=-\iint P_{0} \cos \varphi \cos \theta d \Omega \\
F_{z o}=-\iint P_{0} \cos \theta d \Omega \\
T_{0}=\iint r \sin \theta \times\left(-\frac{H_{0}}{2} \cdot \frac{\partial P_{0}}{r \sin \theta \partial \varphi}-\frac{\mu \omega r \sin \theta}{H_{0}}\right) d \Omega
\end{array},\right.
$$

where: $d \Omega=\left(R^{2} \sin \theta\right) d \varphi \cdot d \theta$. 


\section{Kinetic model for flow field of air gap in maglev spherical active joint}

Due to effects from mass centre and external forces, the rotor rotating at a high speed could suffer turbulence at its balanced position. The dynamic response of air film affects the stability of rotor, so it is necessary to study its dynamic characteristics.

4.1. Dynamic pressure distribution model for air circulation in air gap of maglev joint

Under turbulence, the rotor will deviate from its balanced point, where the three coordinate components of the turbulence are represented by $E_{x 0}, E_{y 0}$ and $E_{z 0}$, respectively. Assuming the turbulence appears in a sinusoidal form, the rotor position at any time can be expressed as [18]:

$$
\left\{\begin{array}{l}
\varepsilon_{x}=\varepsilon_{x 0}+E_{x 0} e^{i f t} \\
\varepsilon_{y}=\varepsilon_{y 0}+E_{y 0} e^{i f t}, \\
\varepsilon_{z}=\varepsilon_{z 0}+E_{z 0} e^{i f t}
\end{array}\right.
$$

where: $f$ is the dimensionless turbulence frequency, $f=\frac{v}{\omega}$, $v$ is the turbulence frequency of rotor; $i$ is the imaginary unit. So we can obtain the thickness of air film for the rotor at any position:

$$
H=H_{0}-H_{d},
$$

where: $H_{0}$ is the thickness of static air film and $H_{d}$ is the thickness of dynamic air film.

$$
\left\{\begin{array}{l}
H_{0}=1-\varepsilon_{x} \sin \theta \cos \varphi-\varepsilon_{y} \sin \theta \sin \varphi-\varepsilon_{z} \cos \theta \\
H_{d}=H_{d 0} \exp ^{i f t}=\left(E_{x} \sin \theta \cos \varphi+E_{y} \sin \theta \sin \varphi+E_{z} \cos \theta\right) \exp ^{i f t}
\end{array},\right.
$$

where: $H_{d 0}$ is the amplitude of dynamic air film, a complex number.

Under slight turbulence, the pressure of dynamic air film can be expressed as:

$$
\begin{aligned}
P=P_{0}+P_{d}= & P_{0}+P_{d 0} \exp ^{i f t}, \\
& \frac{\partial}{\partial \varphi}\left(P_{0} H_{0}^{3} \frac{\partial P_{d o}}{\partial \varphi}\right)+\sin ^{2} \theta \frac{\partial}{\partial \theta}\left(P_{0} H_{0} \frac{\partial P_{d 0}}{\partial \theta}\right)+\sin \theta \cos \theta\left(P_{0} H_{0}^{3} \frac{\partial P_{d 0}}{\partial \theta}\right)+\frac{\partial}{\partial \varphi}\left(H_{0}^{3} \frac{\partial P_{0}}{\partial \varphi} P_{d 0}\right)+ \\
& +\sin ^{2} \theta \frac{\partial}{\partial \theta}\left(H_{0}^{3} \frac{\partial P_{0}}{\partial \theta} P_{d 0}\right)+\sin \theta \cos \theta\left(H_{0}^{3} \frac{\partial P_{0}}{\partial \theta} P_{d 0}\right)+\frac{\partial}{\partial \varphi}\left(3 H_{0}^{2} P_{0} \frac{\partial P_{0}}{\partial \varphi} H_{d 0}\right)+ \\
& +\sin ^{2} \theta \frac{\partial}{\partial \theta}\left(3 H_{0}^{2} P_{0} \frac{\partial P_{0}}{\partial \theta} H_{d 0}\right)+\sin \theta \cos \theta\left(3 H_{0}^{2} P_{0} \frac{\partial P_{0}}{\partial \theta} H_{d 0}\right)= \\
& =\Lambda \sin ^{2} \theta \frac{\partial}{\partial \varphi}\left(H_{0} P_{d 0}+P_{0} H_{d 0}\right)+i 2 \Lambda f \sin ^{2} \theta\left(H_{0} P_{d 0}+P_{0} H_{d 0}\right) .
\end{aligned}
$$

This dynamic equation of air film (14) implies the turbulence components $E_{x 0}, E_{y 0}$ and $E_{z 0}$. In order to obtain the dynamic stiffness and damping coefficient of the air film under dynamic pressure, the partial derivative method is used. Let: $\quad P_{E x}=\frac{\partial P_{d 0}}{\partial E_{x 0}}, \quad P_{E y}=\frac{\partial P_{d 0}}{\partial E_{y 0}}, \quad P_{E z}=\frac{\partial P_{d 0}}{\partial E_{z 0}}$,

$$
H_{E x}=\frac{\partial H_{d 0}}{\partial E_{x 0}}, H_{E y}=\frac{\partial H_{d 0}}{\partial E_{y 0}}, H_{E z}=\frac{\partial H_{d 0}}{\partial E_{z 0}} .
$$

Finding the derivative of Eq. (14) and $H_{d 0}$ gives the equations for $P_{E x}, P_{E y}$, and $P_{E z}$. Equations for $P_{E x}$ are:

$$
\begin{aligned}
& \sin ^{2} \theta \frac{\partial}{\partial \theta}\left(P_{0} H_{0} \frac{\partial P_{E x}}{\partial \theta}\right)+\frac{\partial}{\partial \varphi}\left(P_{0} H_{0}^{3} \frac{\partial P_{E x}}{\partial \varphi}\right)+\sin \theta \cos \theta\left(P_{0} H_{0}^{3} \frac{\partial P_{E x}}{\partial \theta}\right)+\sin ^{2} \theta \frac{\partial}{\partial \theta}\left(H_{0}^{3} \frac{\partial P_{0}}{\partial \theta} P_{E x}\right)+ \\
& +\frac{\partial}{\partial \varphi}\left(H_{0}^{3} \frac{\partial P_{0}}{\partial \varphi} P_{E x}\right)+\sin \theta \cos \theta\left(H_{0}^{3} \frac{\partial P_{0}}{\partial \theta} P_{E x}\right)+3 H_{0}^{3} P_{0}\left[\frac{\partial P_{0}}{\partial \varphi} \frac{\partial}{\partial \varphi}\left(\frac{1}{H_{0}} H_{E x}\right)+\sin ^{2} \theta \frac{\partial P_{0}}{\partial \theta} \frac{\partial}{\partial \theta}\left(\frac{1}{H_{0}} H_{E x}\right)\right]+ \\
& +3 \Lambda \sin ^{2} \theta \frac{1}{H_{0}} \frac{\partial\left(P_{0} H_{0}\right)}{\partial \varphi} H_{E x}=\Lambda \sin ^{2} \theta \frac{\partial}{\partial \varphi}\left(H_{0} P_{E x}+P_{0} H_{E x}\right)+i 2 \Lambda f \sin ^{2} \theta\left(H_{0} P_{E x}+P_{0} H_{E x}\right)
\end{aligned}
$$

$$
H_{E x}=\cos \varphi \sin \theta
$$

Then solve for the boundary conditions:
1. At the place where the air gap contacts with the atmosphere, $P_{0}=P_{a}$;

2. The rotor has an eccentric angle 
$\varphi_{0}=\arctan \left(\varepsilon_{y} / \varepsilon_{x}\right)$ and let $P_{0}=P_{a}$ at the place where $\varphi=\arctan \left(\varepsilon_{y} / \varepsilon_{x}\right)+\pi$.

We can combine with Eq. (15) to obtain $P_{E x}$. Then, $P_{E y}$ and $P_{E z}$ can be obtained in the same way.

4.2. Dynamic characteristic parameters for flow field of air

$$
\begin{aligned}
& {\left[\begin{array}{lll}
K_{x x} & K_{x y} & K_{x z} \\
K_{y x} & K_{y y} & K_{y z} \\
K_{z x} & K_{z y} & K_{z z}
\end{array}\right]=-\iint\left[\begin{array}{c}
P_{E x r} \sin \theta \cos \varphi \\
P_{E x r} \sin \theta \sin \varphi \\
P_{E x r} \cos \theta
\end{array}\right.} \\
& {\left[\begin{array}{lll}
C_{x x} & C_{x y} & C_{x z} \\
C_{y x} & C_{y y} & C_{y z} \\
C_{z x} & C_{z y} & C_{z z}
\end{array}\right]=-\iint\left[\begin{array}{c}
P_{E x i} \sin \theta \cos \varphi \\
P_{E x i} \sin \theta \sin \varphi \\
P_{E x i} \cos \theta
\end{array}\right.}
\end{aligned}
$$

\section{Simulation and experimental study for static $\&$ dynamic performances of flow field of air gap in maglev spherical active joint}

Write a program via finite difference method based on above analysis results to calculate the static \& dynamic

\section{gap in maglev joint}

By obtaining the pressure $P_{0}$ of static air film and substituting it into the dynamic equation of air film, we can obtain the dynamic pressure distribution of air film. With $P_{E x}, P_{E y}$ and $P_{E z}$ known, we can obtain the dimensionless dynamic stiffness and the dynamic damping coefficient, expressed as follows, respectively:

$$
\left.\begin{array}{cc}
P_{E y r} \sin \theta \cos \varphi & P_{E z r} \sin \theta \cos \varphi \\
P_{E y r} \sin \theta \sin \varphi & P_{E z r} \sin \theta \sin \varphi \\
P_{E y r} \cos \theta & P_{E z r} \cos \theta
\end{array}\right] r^{2} d \varphi d \theta, .
$$

performances of air film in the spherical joint. By investigating how the eccentric position and speed of the rotor affect the static pressure and damping moment of the air film, as well as how the turbulence frequency of rotor influences the dynamic stiffness and damping coefficient of the air film, we can obtain the parameters of spherical joint and its operation parameters, as listed in Table 1.

Table 1

Parameters of spherical joint

\begin{tabular}{|c|c|c|c|c|c|}
\hline Rotor radius & Radius gap & Ambient pressure & Dynamic viscosity of air & Angular frequency for rotor working & Number of bearings \\
\hline$r / \mathrm{mm}$ & $c / \mathrm{mm}$ & $p / \mathrm{Pa}$ & $\mu /(\mathrm{Pa} \cdot \mathrm{s})$ & $\omega /\left(\mathrm{r} \cdot \mathrm{min}^{-1}\right)$ & $\Lambda$ \\
\hline 38.5 & 0.3 & $1.013 \times 10^{5}$ & $1.8 \times 10^{-5}$ & $2 \times 10^{5}$ & 3.5 \\
\hline
\end{tabular}

\subsection{Performance simulation for flow field of air gap in maglev spherical active joint}

Fig. 6 illustrates the distribution pattern of loaded pressure for the flow field of air gap in joint. The loaded pressure of the flow field of air gap would be in proportion to the load-carrying stiffness of the air gap if a controller is used to maintain the air gap. Therefore, analysing the variation pattern of load-carrying capacity for the air gap could manifest the variance tendency of the air film stiffness. Many factors, mainly the average length of air gap, the speed of rotor and the eccentricity, can impact the distribution pattern of load-carrying capacity or stiffness of air film in the flow field of air gap.

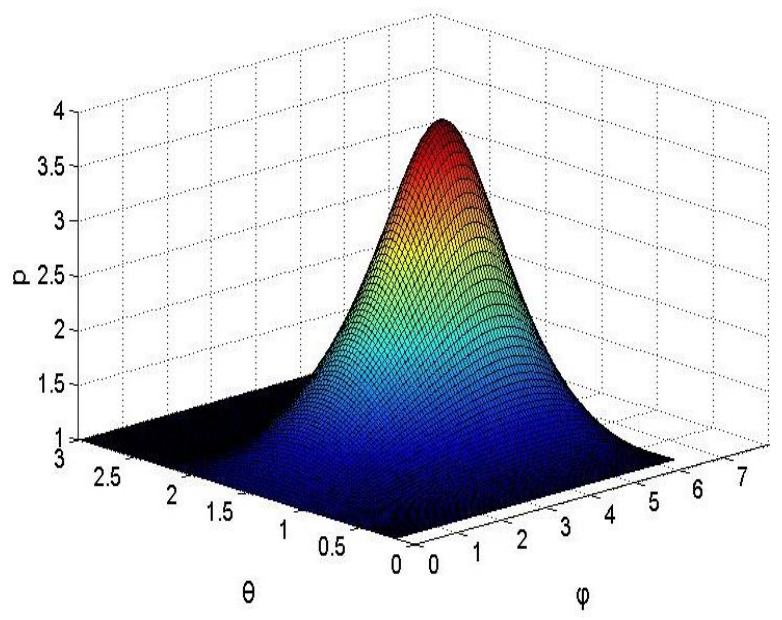

How the load-carrying capacity of air film varies with the average length of air gap is shown in Fig. 7, where the load-carrying capacity drops as the average length goes up. The reason is that the load-carrying capacity will exponentially drop as the average length goes up.

Fig. 8 shows the variation of the load-carrying capacity of air film with the angular velocity of rotor, where it can be found both the load-carrying capacity and the stiffness increase as the rotation speed increases, because the load-carrying coefficient of air film as the rotation speed increases. However, since the film is communicated to the atmosphere, the load-carrying capacity and the stiffness cannot increase endlessly but their variances tend to be gentle in the end.

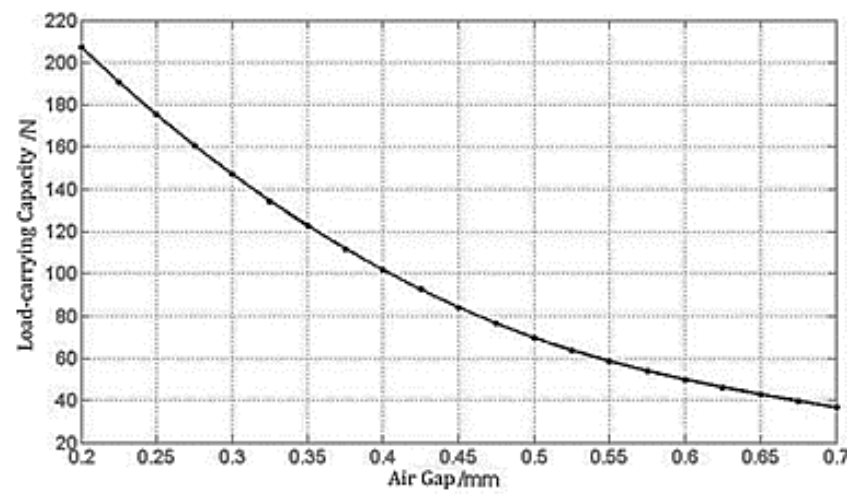

Fig. 7 Variation of load-carrying capacity of air film

Fig. 6 Loaded pressure distribution for the air film 




Fig. 8 Variation of load-carrying capacity of air film with angular velocity of rotor

Fig. 9 exhibits the variation of the load-carrying capacity of air film with the eccentricity, where the load-carrying capacity significantly increases as the eccentricity goes up. The reason is that once the film thickness $h$ at a position changes, the film pressure will alter correspondingly to maintain continuity.

Providing the rotation speed of joint is 100000 $\mathrm{r} / \mathrm{min}$ and the turbulence displacement in positive $\mathrm{x}$-axis is $1 \mu \mathrm{m}$, three contour plots, as shown in Fig. 10, can be obtained to illustrate the dynamic pressure change of air film when the air gap in z-axis lower spherical surface of joint is set at $0.5 \mathrm{~s}, 2 \mathrm{~s}$ and $5 \mathrm{~s}$, respectively. According to simulation results, the dynamic pressure zone of the air gap keeps changing with the rotation of rotor, and the film pressure under turbulence displacement is higher than that under static balance, which is because the turbulence displacement increases the eccentricity, enabling the film pressure to be increased as well. Based on the colour distribution in these plots, the pressure around $\mathrm{z}$-axis approximately shows a sinusoidal (or cosine) distribution.



Fig. 9 Variation of load-carrying capacity of air film with eccentricity

Fig. 11 shows the dynamic stiffness diagram and the dynamic damping coefficient diagram of air film when the eccentricity is set to $\varepsilon_{x}=0.4, \varepsilon_{y}=0.4$ and $\varepsilon_{z}=-0.6$.

We can see from Fig. 11 that the dynamic stiffness and the damping coefficient show close relations with the turbulence frequency of rotor. Generally, when $f<2$, as the turbulence turns more frequent, dynamic stiffness $K_{x x}, K_{y y}$ and $K_{z z}$ increase, while the absolute values of $K_{x y}, K_{x z}, K_{y x}$, $K_{y z}, K_{z x}$ and $K_{z y}$ decrease. All absolute values of dynamic damping coefficients decline.

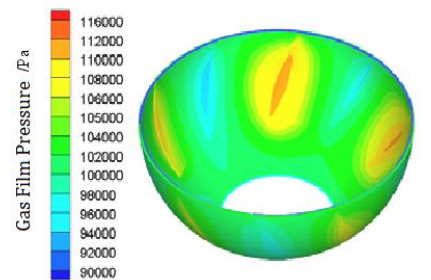

a

c

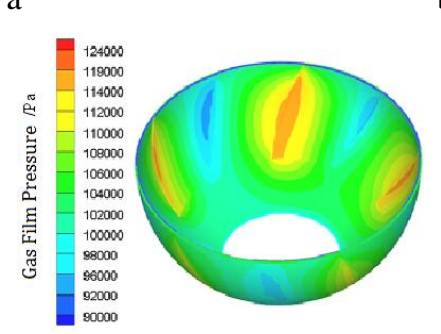

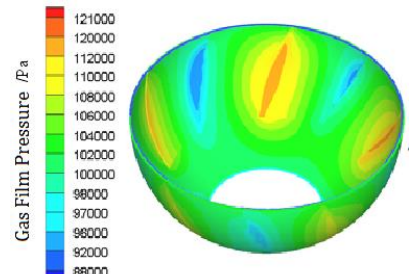

b
Fig. 10 Variation of dynamic pressure in flow field of air gap with rotation: $\mathrm{a}-0.5 \mathrm{~s}, \mathrm{~b}-2 \mathrm{~s}, \mathrm{c}-5 \mathrm{~s}$

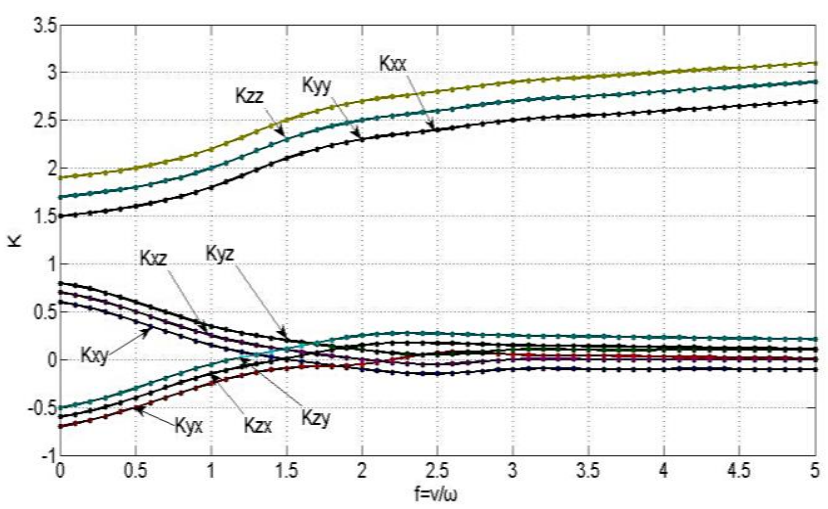

a

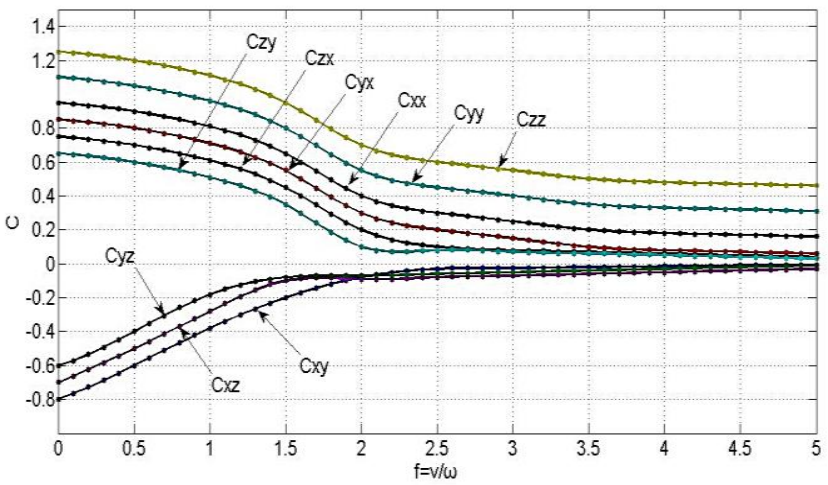

b

Fig. 11 Dynamic characteristics for flow field of air gap: a - dynamic stiffness of air film, $b$ - damping coefficient of air film

5.2. Experiment for load-carrying capacity of flow field of air gap

Fig. 12 shows an experiment setup for controlling an inductive maglev spherical active joint studied in this paper, and this setup was consisted of a joint, an input channel for data collected in the experiment, an output channel for control, and a high-speed processor. 
First, the experiment setup as shown in Fig. 12 was used to measure the resultant supporting force of joint's rotor. Then, subtract the measured electromagnetic levitation force from the resultant supporting force according to Newton's second law, and the obtained result was approximately equal to the force by which the air film in the flow field of air gap supported the rotor rotating at a high speed. we only compared the abovementioned theoretical simulation results with the experimental results in terms of variation pattern or tendency. When the rotor of the maglev spherical active joint rotated at a high speed around the z-axis, the air circulation pressure distribution pattern in the flow field of air gap, which was located in the lower hemispherical surface of joint's stator and vertical to the spindle and passed through the section with maximum load, is shown in Fig. 13. Its distribution pattern and the air film pressure simulation results are similar to those shown in Fig. 10. The air circulation pressure distribution pattern mainly was dependent on $\frac{c}{r}$, the ratio between air gap length (clearance) and rotor radius. As shown in the figure, when the gap was small, the air film acted as a fluid plain bearing and the Reynolds number effect occurred. But when the ratio increased, the Bernoulli effect arose, which caused the rotor to vibrate and displace, leading to increased flow rate and decreased pressure at the side where the gap decreased. As the vibration displacement increased further, the rotor in vibrating would lose its stability, thus determining the boundary condition for the stability of vibration of rotor [19].

$$
\omega_{c} \leq 2 \omega_{n}
$$

Where: $\omega_{c}$ is the critical speed of the rotor losing stability; and $\omega_{n}$ is the intrinsic frequency of rotor.

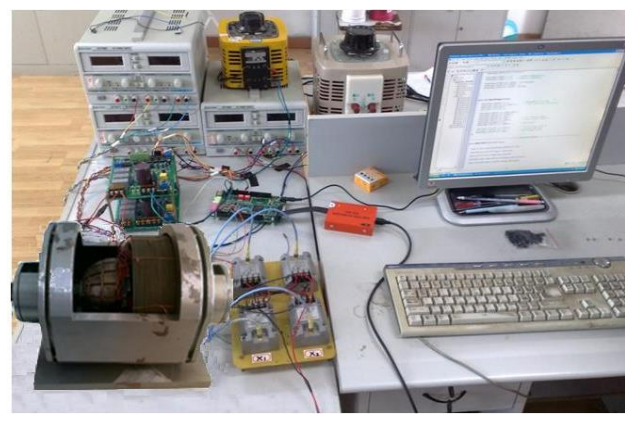

Fig.12 Control system for joint prototype

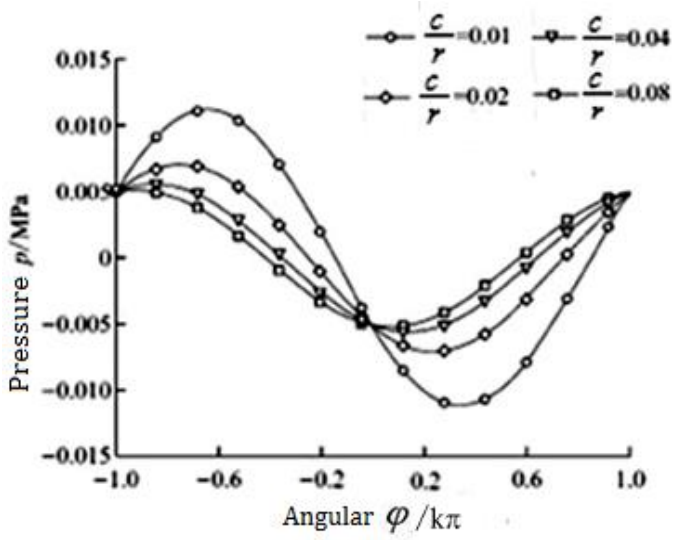

Fig. 13 Pressure distribution for air circulation in air gap

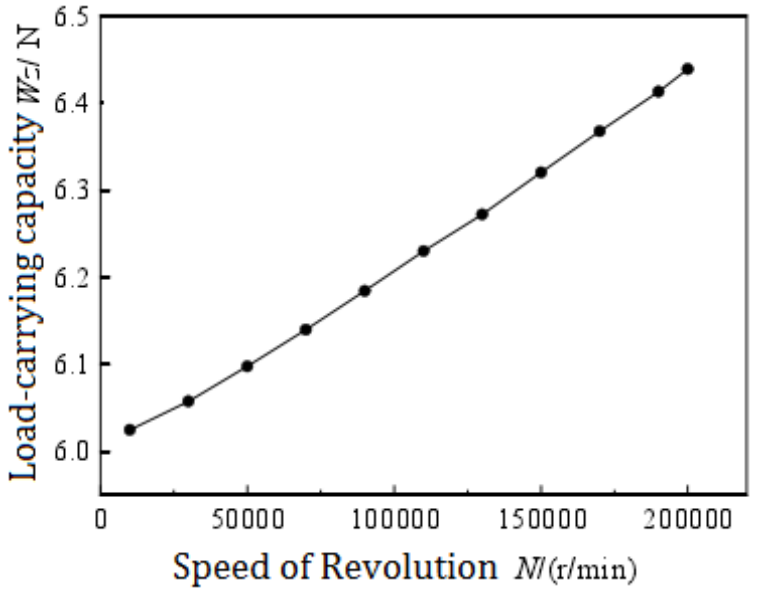

a

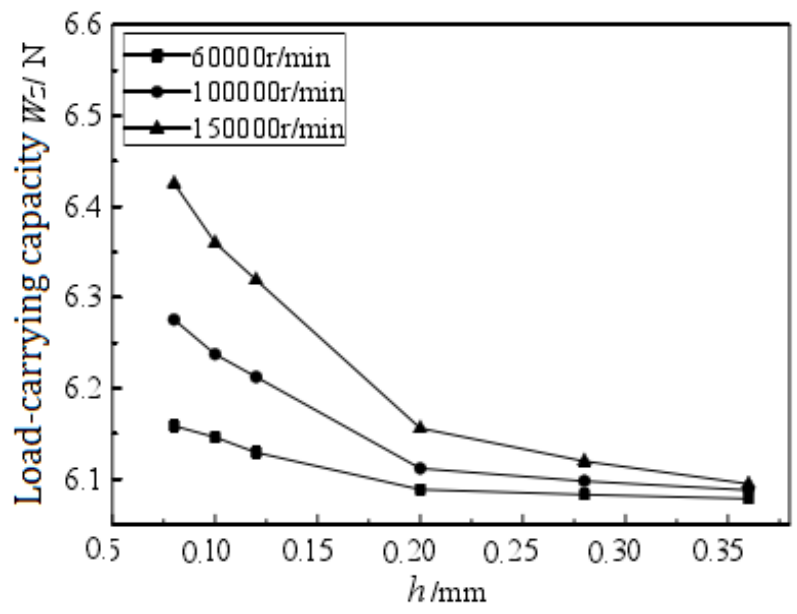

b

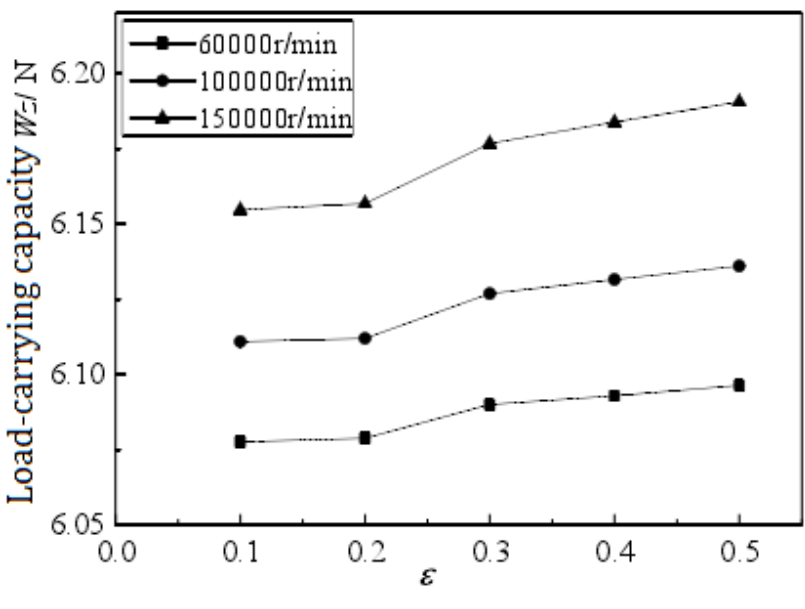

Fig. 14 Factors affecting the load-carrying capacity of air film: a - Load-carrying capacity changes with speed, b - Load-carrying capacity changes with gap, c - Load-carrying capacity changes with eccentricity

Fig. 14 reflects major factors affecting the loadcarrying capacity of air film. Fig. 14, a shows how the loadcarrying capacity of air film changes with the rotation speed in general, the capacity increases as the speed increases, with the variation tendency and the simulation analysis results as shown in Fig. 7. However, due to restricted practical test conditions and test errors, there was little consistency 
between test data and theoretical data. Fig.14, b represents how the load-carrying capacity of air film changes with the air gap under different rotation speeds of the joint, where it can be found that, the shown tendency of the radial loadcarrying capacity changing with the air gap under different rotation speeds is basically consistent with the theoretical simulation analysis results shown in Fig. 7 i.e. the capacity drops as the air gap increases.

Fig. 14, c shows how the load-carrying capacity of air film changes with the eccentricity under different rotation speeds of joint. It can be found in this figure that regardless of the speed of joint, all curves show basically the same variation pattern, which is generally consistent with the theoretical simulation analysis results. As eccentricity goes up, the load-carrying capacity of air film increases and its hydrodynamic effect turns more significant, which can benefit the operation performance of joint.

\section{Conclusions}

Since a machine spindle requires multi-DOF movement, this paper proposes a multi-DOF maglev spherical active joint, and analyses the three-dimensional rotation of active joint rotor around any axis of a fixed spatial point, as well as the principle of maglev fields. It also established the mathematical models for static \& dynamic pressures and dynamic parameters of air gap flow field in the maglev spherical active joint, based on Reynold's equation of dynamic pressure of flow field air. In addition, simulation and experimental study are performed on air film's load-carrying capacity in the air gap flow field, as well as its dynamic characteristics. The experimental and simulation results are qualitatively compared to validate the effectiveness of air gap flow field model, with discussions on the main factors affecting the joint's load-carrying capacity. The research shows that in the drive application of multi-DOF active joint, the influence of air gap flow field is ignored, but its effect grows more significant with the increase of spindle rotor speed. Therefore, the research in this paper provides a new idea and method to make better use of 6-DOF maglev spherical active joint in spindle research and development, and to explore the dynamic characteristics of high-speed spindle and the use of pneumatic structure.

\section{Acknowledgments}

This work was financially supported by China Natural Science Foundation under Grant No. 51375427, Jiangsu Natural Science Foundation under Grant No. BY201411708 and No. BY2015061-04, Jiangsu Industry-UniversityResearch Foundation for Prospective Joint Study under Grant No. BY2014117-08 and No. BY2015 061-04, and Yangzhou City-University-Cooperation Research Foundation under Grant No. YZ2016255. The support is gratefully acknowledged.

\section{References}

1. Changhe, Li; Guangqi, Cai. 2008. Development and research status of parallel machine tool, Journal of Qingdao Technological University, 29(1): 7-13. (in Chinese).
2. Li, Z.; Lun, Q.; Xing, D.; Gao, P. 2015. Analysis and implementation of a 3-DOF deflection-type PM motor, IEEE Transactions on Magnetics, 51(11): 1-4. https://doi.org/10.1109/TMAG.2015.2448534.

3. Kim, H. Y.; Kim, H.; Gweon, D. G.; Jeong J. 2015. Development of a novel spherical actuator with two degrees of freedom, IEEE/ASME Transactions on Mechatronics, 20(2): 532-540.

https://doi.org/10.1109/TMECH.2014.2308417.

4. Li, H.; Shen, Y. 2015. Thermal analysis of the permanent-magnet spherical motor, IEEE Transa-ctions on Energy Conversion, 30(3): 991-998. https://doi.org/10.1109/TEC.2015.2419636.

5. Tsukano, M.; Hirata, K.; Maeda, S.; Niguchi, N. 2015. Development of control method for outer-rotor spherical actuator, Electrical Engineering in Japan, 194(2): 54-63. https://doi.org/10.1002/eej.22729.

6. Kasashima, N.; Ashida, K.; Yano, T.; Gofuku, A.; Shibata, M. 2016. Torque control method of an electromagnetic spherical motor using torque map, IEEE/ASME Transactions on Mechatronics, 21(4): 2050-2060. https://doi.org/10.1109/TMECH.2016.2541679.

7. Kun, Bai; Ruoyu, Xu; Kok-meng, Lee; Wang, Dai; Yongan, Huang. 2018. Design and development of a spherical motor for conformal printing of curved electronics, IEEE Transactions on Industrial Electronics, 65(11): 69-75.

8. Wang, Kang; Wang, Dong; Wu, Leitao; Su, Zhenzhong; Zhang, Xianbiao; Chen, Junquan. 2015. Dynamic stiffness of the permanent magnet biased axial magnetic bearing with multiple air gaps considering eddy current effect, Electric Machines \& Control Application, 42(12): 17-22, 30.

9. Antunes, J.; Axisa, F.; Grunenwald, T. 1996. Dynamics of rotors immersed in eccentric annular flow, Part 1: Theory, Fluids and Structures, 10(8): 893-918. https://doi.org/10.1006/jfls.1996.0058.

10. Fritz, R. J. 1970. The effects of an annular fluid on the vibrations of a long rotor, ASME Journal of Basic Engineering, 92(4): 923-929. https://doi.org/10.1115/1.3425165.

11. Grunenwald, T.; Axisa, F.; Bennett, G., et al. 1996. Dynamics of rotors immersed in eccentric annular flow, Part 2, Experiments, Fluids and Structures, 10(8): 919944. https://doi.org/10.1006/jfls.1996.0059.

12. Fritz, R. J. 1972. The effect of liquids on the dynamic motions of immersed solids, Journal of Engineering for Industry, 94(1): 167-173. https://doi.org/10.1115/1.3428107.

13. Cheng, De; Yao, Zhenqiang; Xue, Yabo; Shen, Hon. 2014. Dynamic behavior of a rotating shaft immersed in a fluid annulus, Journal of Shanghai Jiaotong University, 48(2): 271-276. (in Chinese).

14. Sun, Qiguo; Yu, Lie. 2000. Study of dynamic characteristics for fluid machine rotor immersed in annular liquid flow, Journal of Power Engineering, 20(5): 906-910. (in Chinese).

15. Jiang, Qinglei; Xing, Guikun; Wu, Dazhuan; Wang, Leqin. 2012. Computation of transient fluid induced force of small clearance flow in centrifugal pump, Journal of Zhejiang University, 46(5): 929-934. (in Chinese). 
16. Shi, Minglin; Wang, Dezhong; Zhang, Jige. 2012. Dynamic modeling and analysis of Jeffcott rotor-annular flow coupled system, Journal of Shanghai Jiaotong University, 46(9): 1503-1508(in Chinese).

17. Baoguo, Wang; Fengxiang, Wang. 2002. Excitation and control analysis of levitation force winding for magnetic suspension bearing less motors, Proceedings of the Chinese Society for Electrical Engineering, 22(5): 105108. (in Chinese).

18. Dongli, Qin. 2009. Research on design method and error analysis of a spherical gas-floated and driven gyroscope, Harbin Institute of Technology. (in Chinese).

19. Dazhuang, Qu; Le, Jin; Quan, Zhou; Mengqi, Li; Xin, Jia. 2015. Effects of annular flow on rotor vibration of nuclear power RCP motors, Journal of Chinese Society of Power Engineering, 12(12): 1023-1029. (in Chinese).
Fan Zhang, Li Zeng, Yan Li, Zi Zeng, Zhida Zhu, Jin Sun, Min Dai

\section{A STUDY ON CHARACTERISTICS OF FLOW FIELD \\ OD AIR GAP IN MAGLEV SPHERICAL ACTIVE JOINTS FOR MULTI-DOF SPINDLE SYSTEM}

\section{S u m m a r y}

Realizing six-degree-of-freedom movement for spindle tools in any posture is an important research direction for CNC machines. Existing spindle systems with parallel or cascaded structure, due to their complex mechanical structure, lead to serious abrasion on mechanical supports when rotating at a high or ultra-high-speed, which heats joint parts and worsens the dynamic characteristics. In light of technologies of motor and magnetic levitation, this paper proposed a multi-degree-of-freedom machine spindle system built on a maglev spherical active joint, and analyzed working principles of a three-dimensional rotating magnetic field generated by the rotor of maglev spherical active joint rotating around an arbitrary axis passing through a fixed spatial point. This paper also explored the effects of flow field of air gap formed in the maglev spherical active joint on the dynamic \& static characteristics of joint based on aerodynamics, established mathematical models for the dynamic \& static pressures and kinetic parameters of flow field of air gap, and, with simulation and experiment, investigated how the flow field of air gap impacts the static load-carrying capacity and the dynamic characteristics of joint.

Keywords: multi-degree-of-freedom spindle; maglev active joint; flow field of air gap; pressure distribution; loadcarrying capacity; dynamic characteristics.

Received February 13, 2018

Accepted October 18, 2018 